\title{
Set-invariance analysis for deterioration prediction on a roller-on-tire actuator
}

\author{
Diego Rodriguez-Obando, John J. Martinez and Christophe Berenguer
}

\begin{abstract}
The paper deals with the problem of predicting deterioration of a roller-on-tire actuator in presence of unpredictable but bounded motor torques. Set-invariance is used to characterize the nominal behavior of the actuator and the maximal admissible deterioration behavior. A novel index of deterioration is presented and allows to model the mechanical actuator as a polytopic Linear Parameter Varying model. For a given initial deterioration, the probabilistic certification approach is used to numerically predict an interval of "expected time" for reaching the total outage of the actuator. A simulated example illustrates the interest of the proposed approach and its potential integration on future predictive maintenance techniques.
\end{abstract}

\section{INTRODUCTION}

Prognosis of remaining useful lifetime (RUL) is a widely studied topic in health management of resources for automation and mechanics [10], [4]. Most research works about RUL focus on preventive maintenance applications [13], [3]. Nowadays manufacturers are becoming increasingly motivated to manage the complete lifecycle of an asset and to optimize the production process and its energy consumption using proactive maintenance strategies and "RUL-aware" operation of their system. Then, the design of new prognostic models of deterioration is a necessary to implement the maintenance in the proactive level. The RUL prediction is not only necessary to verify if the mission goal can be accomplished but also important to aid in online decision-making activities such as fault mitigation, mission replanning, maintenance, etc.

This paper presents a model-based approach for estimation of deterioration in a roller-on-tire actuator. Several motion applications are based on friction. This work is related to actuators based on friction between two rotatory devices. Friction rollers, railway wheels, and friction drive electrical bicycles are examples of this kind of actuators. In practice, both the surfaces of the motor and the driven device will deteriorate and their deterioration reach eventually a threshold above which the system is considered failed.

The presented deterioration model is based on the computation of an image of the dissipated energy at the contact level. A contact quality coefficient is proposed and allows to model the actuator as a (polytopic) Linear Parameter Varying model. In this paper we show that Robustly positive invariant sets can be used to characterizes the nominal and the admissible degraded behavior of the system. This invariant sets

\footnotetext{
${ }^{1}$ D. Rodriguez-Obando, J. Martinez and C. Bereguer are with the Univ. Grenoble Alpes, CNRS, GIPSA-lab, F-38000 Grenoble, France name. surnamedgipsa-lab.fr
}

can be computed using the approach, for polytopic systems, proposed in [8].

Since prognostics deals with predicting the future behavior of engineering systems, there are several sources of uncertainty which influence such future prediction, and therefore, it is rarely feasible to obtain an estimate of the RUL with complete precision. In fact, it is not even meaningful to make such predictions without computing the uncertainty associated with RUL. In this paper a preliminary work related to the use of Probabilistic certification approach, as proposed in [1], is explored and discussed. Given a family of constraints (deterioration produced by bounded inputs), the scenario-based approach, presented here, suggests to simulate $N$ possible trajectories of the deterioration and compute the number of times the constraints were violated. The obtained statistical probability is associated with a confidence parameter, since it is the probability that the success statement is wrong. As stated in [2], we have to compute a sufficiently large number of random trials to guarantee a certain confidence. In this paper, the last section is dedicated to analyze two cases of uncertainties (uncertain input and uncertain parameter cases). It is shown that it is possible to obtain an estimation of an interval of possible RUL, with a certain confidence percentage based on the presented deterioration model. The task associated to this prediction can be started by a condition based on the invariant sets characterizing the admissible deterioration.

The paper is organized as follows: First a roller-on-tire dynamical model is presented in section 2. Section 3 presents a dynamical model of the deterioration of the actuator. The application of robustly positive invariance for solving the problem of detecting an abnormal deterioration is presented in Section 4. Finally, section 5 illustrates two particular cases of uncertainties and the probabilistic certification approach for estimating the Remaining Useful Life of the actuator. The paper ends with Section 6 that summarizes the paper contribution and gives some hints for future investigation.

\section{ROLLER-ON-TIRE ACTUATOR MODEL}

The considered system is a roller-on-tire actuator as it is shown in Figure 1. The symbols used in this work are presented in Table I. Here the force $F_{c}$ is assumed to be a tangential common force for both motor device and driven device (a tire). This force, produced by the motor, causes a torque which drives the wheel. This force will depend on the tangential speeds produced for both motor and wheel, denoted as $v_{1}$ and $v_{2}$ respectively. 
TABLE I

SYMBOLS USED FOR MODELING THE ROLLER-ON-TIRE ACTUATOR

\begin{tabular}{|c|c|c|}
\hline Symbol & Units & Physical meaning \\
\hline$v_{1}$ & {$[\mathrm{~m} / \mathrm{s}]$} & Tangential speed of the motor \\
\hline$v_{2}$ & {$[\mathrm{~m} / \mathrm{s}]$} & Tangential speed of the driven device \\
\hline$\omega_{1}$ & {$[\mathrm{rad} / \mathrm{s}]$} & Angular speed of the motor \\
\hline$\omega_{2}$ & {$[\mathrm{rad} / \mathrm{s}]$} & Angular speed of the driven device \\
\hline$\dot{\omega}_{1}$ & {$\left[\mathrm{rad} / \mathrm{s}^{2}\right]$} & Angular acceleration of the motor \\
\hline$\dot{\omega}_{2}$ & {$\left[\mathrm{rad} / \mathrm{s}^{2}\right]$} & Angular acceleration of the driven device \\
\hline$r_{1}$ & {$[m]$} & External radius of the motor \\
\hline$r_{2}$ & {$[m]$} & External radius of the driven the device \\
\hline$B_{1}$ & {$\left[\mathrm{Kgm}^{2} / \mathrm{s}\right]$} & Viscous damping coefficient of the motor \\
\hline$B_{2}$ & {$\left[\mathrm{Kgm}^{2} / \mathrm{s}\right]$} & Viscous damping coefficient of the driven device \\
\hline$J_{1}$ & {$\left[K_{g m}^{2}\right]$} & Moment of inertia of the motor \\
\hline$J_{2}$ & {$\left[K g m^{2}\right]$} & Moment of inertia of the driven device \\
\hline$T_{L}(t)$ & {$[N m]$} & Load torque observed by the motor \\
\hline$T_{S}(t)$ & {$[N m]$} & Source torque observed by the driven device \\
\hline$K_{m}$ & {$[V \mathrm{~V} / \mathrm{rad}]$} & Motor back-electromotive force constant \\
\hline$I$ & {$[A]$} & Electrical motor current \\
\hline
\end{tabular}

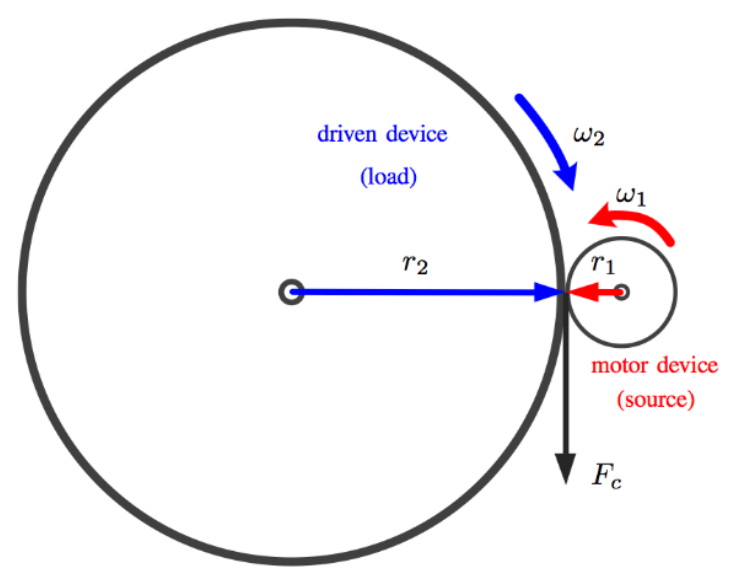

Fig. 1. Roller-on-tire system

Using Newton's laws of motion, the roller-on-tire dynamics can be written as follows:

$$
\begin{aligned}
& J_{1} \dot{\omega}_{1}(t)=-B_{1} \omega_{1}(t)+T_{L}(t)+K_{m} I(t) \\
& J_{2} \dot{\omega}_{2}(t)=-B_{2} \omega_{2}(t)+T_{S}(t)
\end{aligned}
$$

where $T_{L}(t)$ and $T_{S}(t)$ are the torques observed from the motor side and from the driven side, respectively. They can be written in terms of the contact force $F_{c}(t)$ as follows:

$$
\begin{aligned}
& T_{L}(t)=-F_{c}(t) r_{1} \\
& T_{S}(t)=F_{c}(t) r_{2}
\end{aligned}
$$

The contact force $F_{c}(t)$ can be characterized by using dynamic friction models, see for instance [5] and [6]. Due to the fact that in this work we will concentrate the analysis for long periods of time, only macroscopic friction phenomena will be kept for modeling a more simple contact model. Therefore, the main assumption in this paper will be that the contact force is proportional to the relative tangential velocity of the rotational devices at the point of contact. That is,

$$
F_{c}(t)=\alpha\left(v_{1}(t)-v_{2}(t)\right)=\alpha\left(r_{1} \omega_{1}-r_{2} \omega_{2}\right)
$$

\section{A. Modeling the actuator as an Uncertain Linear System}

Defining the system state as $x:=\left[\omega_{1}(t) \omega_{2}(t)\right]^{T}$, the control input $u=I(t)$ (the electrical motor current) and taking the equations (1)-(5), the state space representation of the uncertain linear system will be

$$
\begin{aligned}
\dot{x} & =A(\alpha) x+B u \\
y & =C x
\end{aligned}
$$

where $\alpha$ stands for the uncertain parameter (or the scheduling parameter in the case of a linear parameter varying model interpretation), with matrices:

$$
\begin{gathered}
A(\alpha)=\left[\begin{array}{cc}
\left(-\alpha r_{1}^{2}-B_{1}\right) / J_{1} & \alpha r_{1} r_{2} / J_{1} \\
\alpha r_{2} r_{1} / J_{2} & \left(-\alpha r_{2}^{2}-B_{2}\right) / J_{2}
\end{array}\right], \\
B=\left[\begin{array}{c}
K_{m} / J_{1} \\
0
\end{array}\right]
\end{gathered}
$$

and $C$ an identity matrix. That means that both angular speed of the motor and angular speed of the driven device are measured.

\section{B. Definition of deterioration for roller-on-tires actuators}

Let us consider the "deterioration" as a measurement of the ability of the actuator to transfer power to the load device. The power performed by the motor is transformed into mechanical power on the load side by means of the contact forces (5). In this paper, the parameter $\alpha$, in (5), will be considered as a time-varying parameter which characterizes the quality of the contact (e.g. the inter-surface adhesion and the surface roughness) between both rotational devices. In addition, in this work, we consider that this parameter will monotonically decrease in time for modeling the deterioration of the roller-on-tire actuator. In the sequel, this parameter will be called the contact quality coefficient.

\section{DYNAMICAL MODEL OF DETERIORATION}

\section{A. Dissipation-energy based model of deterioration}

Multiplying (5) by the relative tangential speed $\left(r_{1} \omega_{1}-\right.$ $\left.r_{2} \omega_{2}\right)$, the dissipated power at the contact level can be computed as follows:

$$
P_{c}(t)=\alpha\left(r_{1} \omega_{1}-r_{2} \omega_{2}\right)^{2}
$$

The dissipated energy could be considered as an image of the heat and the material worn at the contact level during traction. This assumption is very similar to the Archard's equation that is more commonly used in railway industry for wear prediction [3], [4]. Thus, an index of the deterioration could be obtained as

$$
D(t):=\int_{0}^{t} P_{c}(t) d t=\int_{0}^{t} \alpha\left(r_{1} \omega_{1}-r_{2} \omega_{2}\right)^{2} d t
$$

In addition, by assumption, the contact quality coefficient $\alpha(t)$ decreases as $D(t)$ increases. That is,

$$
\alpha(t)=-m D(t)+\alpha(0)
$$




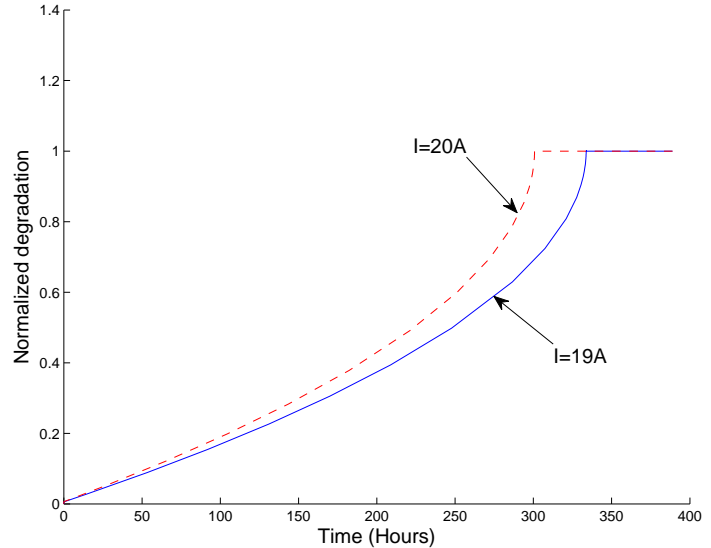

Fig. 2. Normalized deterioration with respect to time, for two different constant electrical currents $(I=19 A$ and $I=20 A), \alpha(0)=10$ and $m=0.01$.

where $m \geq 0$ and $\alpha(0)$ are considered as unknown parameters, but belonging to a given known interval.

Therefore, using (11) and (12) we can compute the dynamics of the parameter $\alpha(t)$, as follows

$$
\dot{\alpha}(t)=-m p(x) \alpha(t)
$$

where $p(x) \geq 0$, called here the sliding factor, is given by

$$
p(x):=\left(r_{1} \omega_{1}-r_{2} \omega_{2}\right)^{2}
$$

Remark that the contact quality deterioration-rate (13), depends on the relative tangential speed, which could be controlled if the uncertain system (6) is controllable, by considering the control input $u=I(t)$.

In terms of the deterioration index $D$, equation (12) can be rewritten in a relative form as follows:

$$
\frac{\alpha(t)}{\alpha(0)}=-\bar{D}(t)+1
$$

where $0 \leq \bar{D}(t) \leq 1$ denotes the normalized deterioration. That is,

$$
\bar{D}(t)=\frac{m}{\alpha(0)} D(t)
$$

Remark that the quality of contact forces $\alpha(t) \rightarrow 0$ as $\bar{D}(t) \rightarrow 1$. Figure 2 illustrates the behavior of $\bar{D}(t)$.

The normalized deterioration can be obtained at every time-instant, by using (15) and the estimations of $\alpha(t)$ and $\alpha(0)$. A suitable state-observer can be designed for this goal. The next subsection establishes the main aspects for designing this observer.

\section{B. Estimating the state of deterioration}

Consider the augmented system:

$$
\begin{aligned}
\dot{x} & =A(\alpha) x+B u \\
\dot{\alpha} & =-m p(x) \alpha \\
\dot{m} & =0 \\
y & =C x
\end{aligned}
$$

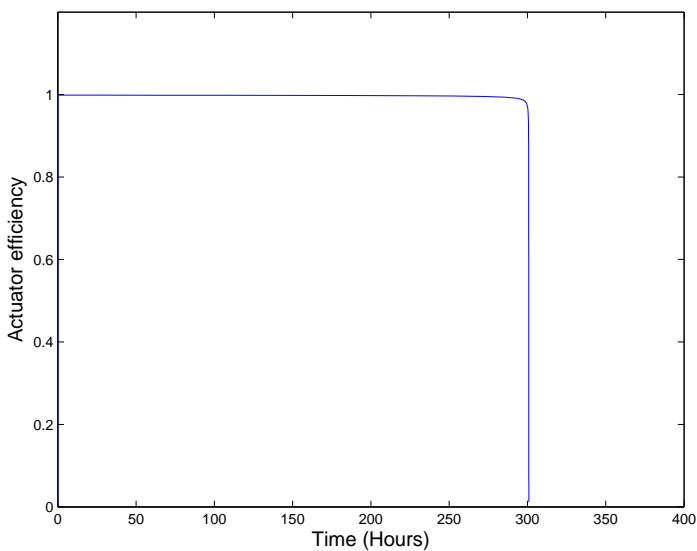

Fig. 3. Efficiency of the actuator $\eta_{a}=\left(r_{2} \omega_{2}\right) /\left(r_{1} \omega_{1}\right)$ for constant input $I=20 A$.

In this paper we assume that this augmented system is observable. Then, it is possible to design an Extended Kalman filter to estimate the states $x$, the contact quality coefficient $\alpha$ and the constant $m$. Because the available measurements are $y=x$, the observability property of this non-linear system mostly depends on the matrix $A(\alpha)$, where $\alpha$ affects the matrix in an affine way.

In the sequel, we assume the availability of the estimations of $\alpha$ and $m$ at every time instant. This means that, using (15), the state of deterioration $\bar{D}$ can be evaluated as well. However, it is not simple to evaluate or estimate, in a reliable way, the Remaining Useful Life (RUL) of the actuator. In other words, the question is, given a deterioration at a particular time-instant, how to estimate the "expected" remaining time, before reaching the total outage of the actuator? The latter question will be explored in section V.

\section{Estimating the efficiency of the actuator}

The instantaneous efficiency of the actuator, denoted $\eta_{a}(t)$, can be estimated at every time-instant by using the measured angular speeds $\omega_{1}(t)$ and $\omega_{2}(t)$. That is,

$$
\eta_{a}(t)=\left(1-\frac{P_{c}(t)}{T_{L} \omega_{1}}\right)=\frac{r_{2} \omega_{2}}{r_{1} \omega_{1}}
$$

This efficiency is a measure of the actual power provided by the motor, at the contact level, and the effective power recovered by the driven device. Remark that this efficiency is independent of the contact quality coefficient $\alpha(t)$. For this reason, the efficiency of the actuator is not a good indicator of the state of the deterioration of the actuator and it does not allow to estimate the RUL. The figure 3 illustrates the obtained efficiency during a total useful life of the actuator. For this scenario, the efficiency is, practically, admissible along the time and only decreases in the moment of the failure (total deterioration), around $t=300$ hours for $I=$ $20 A$.

For the same scenario, the obtained sliding $p(x)$ is illustrated in figure 4. Remark that this function is almost 


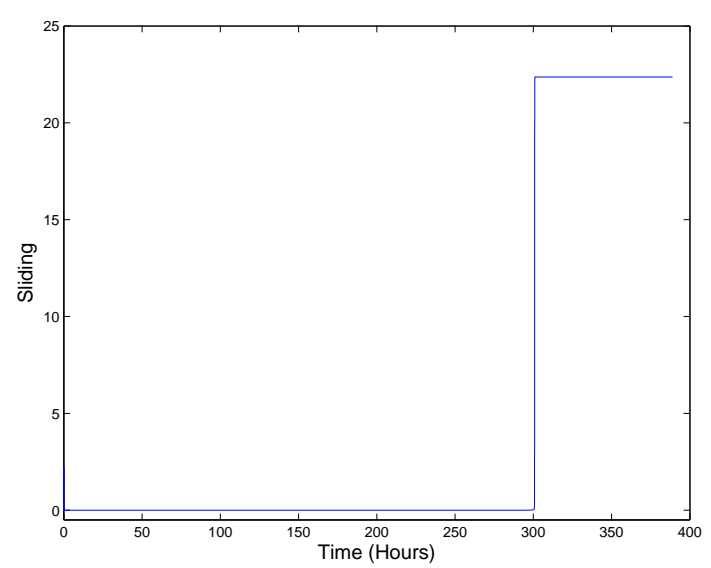

Fig. 4. Sliding $p(x)$ with respect to time in hours.

constant during the total useful life of the actuator and does not depends of the contact quality coefficient $\alpha$. As a consequence, and in the same way than the efficiency of the actuator, the sliding $p(x)$ is not a good indicator of the state of the deterioration of the actuator and it does not allow to estimate the RUL.

\section{INVARIANT SETS FOR DETECTING DETERIORATION}

Starting from the uncertain system (6), consider the discrete-time linear model of the actuator:

$$
x^{+}=A_{d}(\alpha) x+B_{d} u
$$

with $A_{d}(\alpha):=\left(I_{n \times n}+t_{s} A(\alpha)\right)$ and $B_{d}=t_{s} B$, where $t_{s}$ stands by the sampling-time, $I_{n \times n}$ an identity matrix of consistent dimensions, and $x^{+} \in \mathbb{R}^{n}$ is the successor state and $u \in \mathbb{R}^{m}$, the electrical current, is a priori considered as an unknown input (a bounded disturbance).

Because $\alpha$ affects the matrix $A_{d}(\alpha)$ in an affine way, the model (22) can be rewritten in a polytopic form as follows:

$$
x^{+}=\left[\lambda A_{d}(\underline{\alpha})+(1-\lambda) A_{d}(\bar{\alpha})\right] x+B_{d} u
$$

where $0 \leq \lambda \leq 1$. That is, the model (22) can be written as a convex combination of the extreme dynamics which are described by minimal and maximal possible values of $\alpha$, denoted here as $\underline{\alpha}$ and $\bar{\alpha}$, respectively.

The problem now is to compute the minimal robustly positive invariant-set (mRPI set) for system (23), in order to characterize all possible trajectories of the angular speeds (the state $x$ ), at any time and for any sequence of bounded electrical currents $I(t)$. Here we can use the method proposed in [8]. The subsystems, which describe the polytopic system, are assumed to be stable in presence of bounded disturbances and, in addition, to share a common Lyapunov function. Polyhedral RPI sets for linear systems (assuming constant $\alpha$ ) could be also computed using methods such as those proposed in [11], [7], [12] and [9].

Figure 5 depicts the obtained RPI sets for the maximal values of the contact quality coefficient $\alpha$ (i.e. $\bar{\alpha}=\alpha(0)$ ), the white set $\mathcal{S}_{0}$. In addition, the figure 5 illustrates the obtained

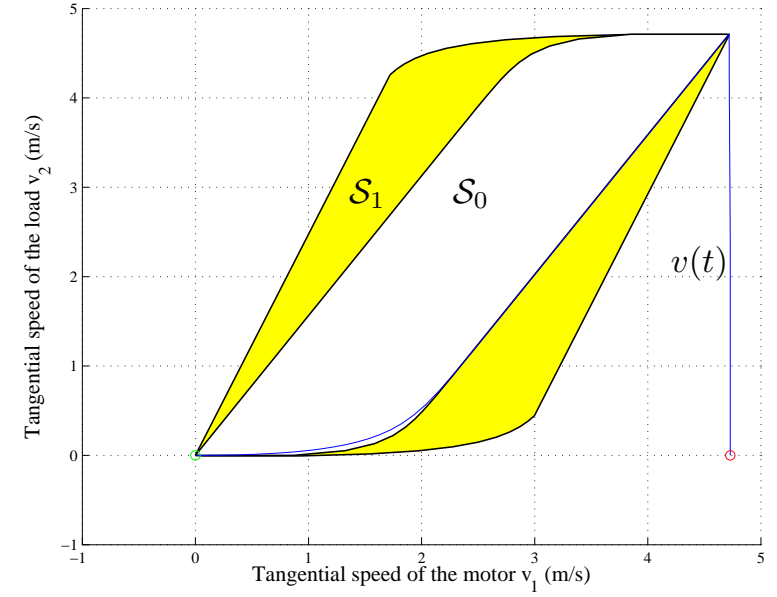

Fig. 5. Robustly positive invariant sets for bounded electrical current $(0 \leq$ $I(t) \leq 20 A)$ and admissible values of $\alpha(\underline{\alpha} \leq \alpha(t) \leq \bar{\alpha})$. A tangential speed $v(t)$ out of these sets means that the actuator deterioration is not more admissible.

RPI set for all "acceptable" values of $\alpha$ (i.e. $\underline{\alpha} \leq \alpha(t) \leq \bar{\alpha}$ ), the set $\mathcal{S}_{1}$. As expected, the latter set $\mathcal{S}_{1}$ includes the set $\mathcal{S}_{0}$. Those sets and the state trajectory, here the tangential speed $v(t):=\left[v_{1}(t) v_{2}(t)\right]^{T}$, can be used for robustly detecting a failure and/or an undesired deterioration of the contact quality.

This approach can be used for starting online decisionmaking activities such as fault mitigation, mission replanning, maintenance, etc. However, the RPI sets do not provide enough information for estimating the Remaining Useful Life (RUL) of the actuator. This point will be explored in the next section.

\section{Estimation of the REMAINING USEFUl LifE}

\section{A. The RUL estimation problem}

The problem is, for a given predefined scenario and/or protocol (fixed duty cycles, minimal and maximal electrical motor current, etc), at every time instant, estimate the RUL of the actuator with a certain precision. It is clear that the estimated RUL has to belong to an interval (a set) which consider the uncertainties of the model and the uncertainties on the realization of the motor current (the exogenous disturbances).

Due to the fact that mechanical equations of motions are well known, the probabilistic certification approach (see for instance [1] and [2]), can aid to estimate (and control) the interval values of the RUL under certain assumptions about the source of uncertainties.

There are two kind of uncertainties that have to be treated here. Uncertainties on the parameters of the model (for instance, the estimation error of the parameter $m$ in (18)) and uncertainties on the topology of the exogenous signals (for instance, the expected scenario for the electrical current $I(t)$ which does not depend on the system state $x$ ). 


\section{B. Uncertain input case}

Figures 6 and 7 illustrate the behavior of the normalized deterioration $\bar{D}(t)$ for a constant electrical current $I(t)=$ $I$. One case considers bounded possible electrical currents $(19 A \leq I \leq 21 A)$, the black lines, and the second case considers random possible electrical currents, the red dotlines. In figure 6 it is considered an initial condition of the normalized deterioration $\bar{D}=0$. The estimated RUL exactly belongs to the time interval $\{272.71,333.88\}$ hours, for the bounded possible currents case. However considering a more realistic scenario (a random possible currents case), the number of actuator population which do not respect this interval is different to zero. In this example, there is a probability that $5 \%$ of the population will deteriorate faster than expected in the bounded current case (i.e. earlier than 272.71 Hours). This estimation was obtained by using $N=$ 233 simulations, with normally distributed pseudorandom values of electrical currents $I(t)$, with mean $20 A$ and a standard deviation of $0.5 \mathrm{~A}$. As suggested in [1] and [2]), the number of simulations $N$ has been computed as follows

$$
N \geq(1 / \eta) *\left(1+\ln (1 / \delta)+(2 \ln (1 / \delta))^{1 / 2}\right)
$$

which guarantee a Confidence parameter $\delta=0.001$ (i.e. $99.9 \%$ of the reliability of the estimation procedure). $\eta$ represents the percentage of actuator population which violate the condition (e.g. $5 \%$ of the population will deteriorate faster than expected).

For an initial condition of the normalized deterioration $\bar{D}=0.4$ as depicted in figure 7 , the estimated $R U L$, computed at the instant 200 hours, belongs to the time interval $\{297.03,319.10\}$ hours. In addition, we obtain the same probability of $5 \%$ of the population will deteriorate faster than expected in the bounded case, with the same confidence parameter $\delta=0.001$.

It is clear that the precision of the estimation of the RUL basically depends on the precision of the estimated $\alpha(t)$ and estimated $m$. Of course, the precision also depends on the quality of the proposed model structure and the assumptions relating the probability distribution of the electrical current $I(t)$.

Figure 8 illustrates the behavior of the deterioration $\bar{D}$ for two different maximal amplitudes of the electrical current, but the same activation sequence, as depicted in figure 9 . Activation sequence is equal to 1 when it is applied the maximal current, and equal to 0 when system is stopped. An interesting observation is that the prediction of the RUL is still quite simple and keeps a certain "invariance" property, providing an interval of possible values of RUL according to the interval of possible maximal electrical currents.

\section{Uncertain parameter case}

Figure 10 illustrates the behavior of the deterioration $\bar{D}$ for 233 random simulations of different values of the parameter $m$. This parameter is assumed to be constant and verifies $0.0095 \leq m \leq 0.0105$. The current sequence is the same, depicted in figure 9. An interesting observation here, is that the behavior of the deterioration is harder to estimate. In

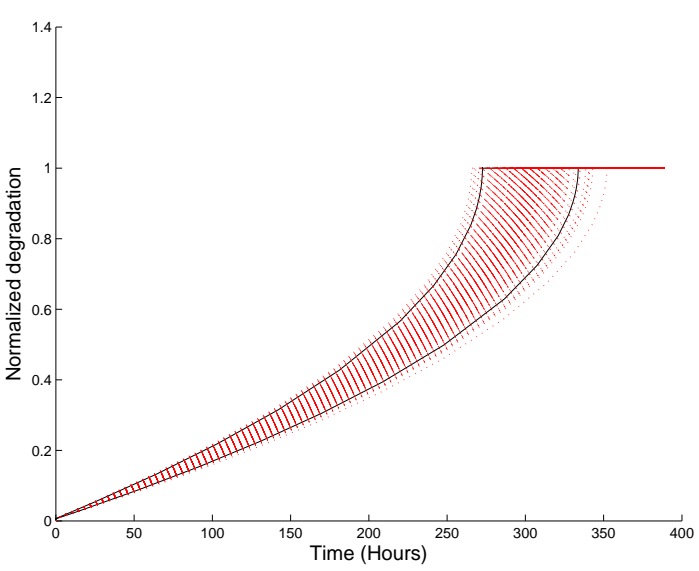

Fig. 6. Estimated deterioration for random $I(t)$, with mean $20 A$ and a standard deviation of $0.5 \mathrm{~A}$. Here, $5 \%$ of the population aging more fast than the (deterministic) bounded current case (i.e. $19 A \leq I(t) \leq 21 A$ ).

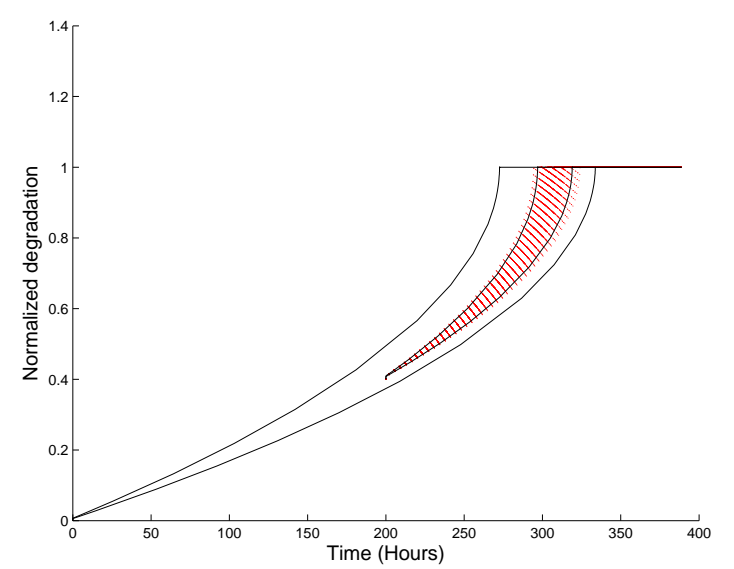

Fig. 7. Estimated deterioration for random $I$, with mean $20 A$ and a standard deviation of $0.5 A$. For an initial condition $\bar{D}=0.4$ at $t=200$ hours. Knowledge of the deterioration at this time allows a more accurate estimation of the remaining time before outage of the actuator.

TABLE II

PARAMETERS OF THE SIMULATED EXAMPLE

\begin{tabular}{lcc}
\hline Parameter & Value & Unit \\
\hline$B_{1}$ & $6.36 \times 10^{-3}$ & {$\left[\mathrm{Kg} \mathrm{m}^{2} / \mathrm{s}\right]$} \\
$B_{2}$ & $1.76 \times 10^{-3}$ & {$\left[\mathrm{Kgm}^{2} / \mathrm{s}\right]$} \\
$J_{1}$ & $3.47 \times 10^{-4}$ & {$\left[\mathrm{Kgm}^{2}\right]$} \\
$J_{2}$ & 0.2 & {$\left[\mathrm{Kgm}^{2}\right]$} \\
$r_{1}$ & 0.0315 & {$[\mathrm{~m}]$} \\
$r_{2}$ & 0.35 & {$[\mathrm{~m}]$} \\
$K_{m}$ & 0.0477465 & {$[V \mathrm{Vs} / \mathrm{rad}]$} \\
$\alpha(0)$ & 10 & - \\
$m$ (nominal) & 0.01 & - \\
\hline
\end{tabular}

particular, the extreme value of $m, m=0.0105$, does not provides the faster deterioration (the black line in figure 10). This aspect clearly justify the use of probabilistic certification as a less conservative way to compute a reliable RUL. Table II presents the parameters values of the performed example. 


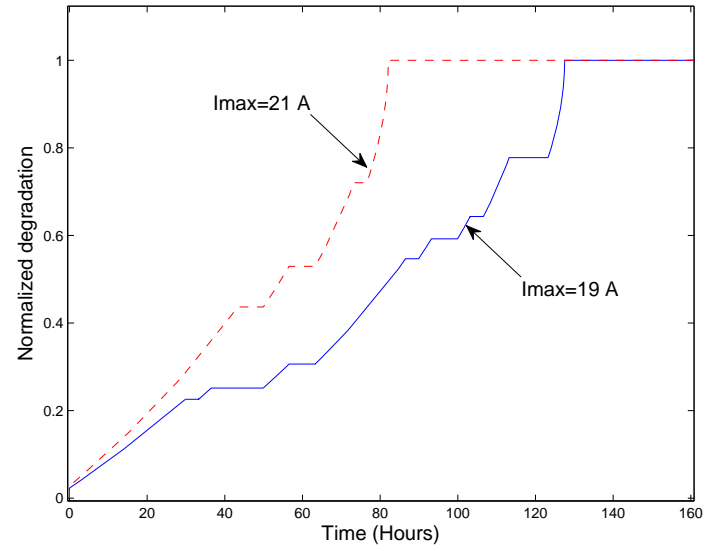

Fig. 8. Deterioration obtained with different maximal electrical currents for a predefined sequence of $u(t)$

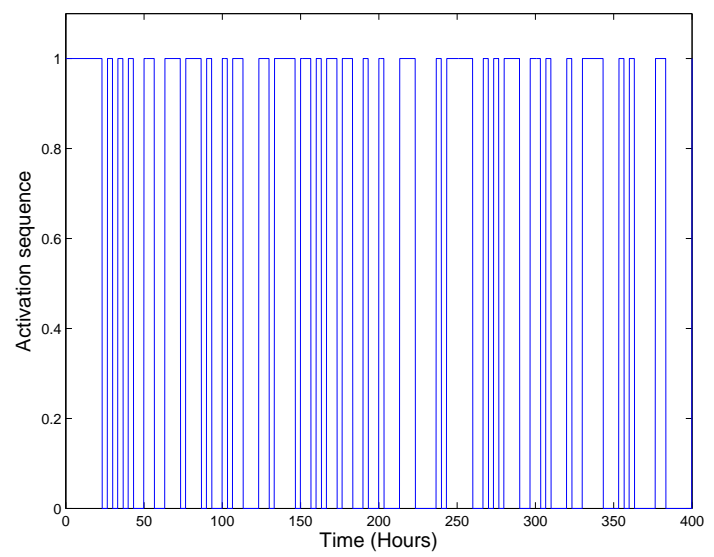

Fig. 9. Activation sequence of signal $u=I(t)$.

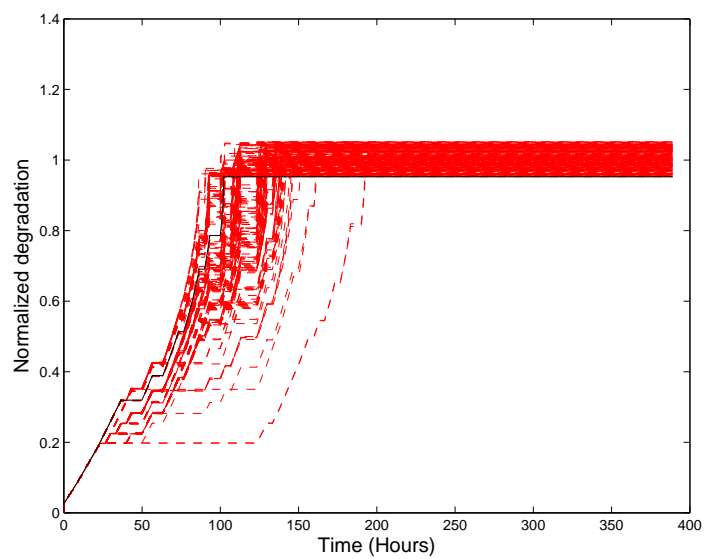

Fig. 10. Deterioration obtained with 233 random simulations for $0.0095 \leq$ $m \leq 0.0105$. The (black line) concerns the curve with the maximal value of $m$.

\section{CONCLUSIONS AND FUTURE WORK}

In this paper we have presented a novel dynamical model of deterioration of a roller-on-tire actuator. A novel index of deterioration (called the contact quality coefficient) is presented and allows to model the mechanical actuator as a polytopic Linear Parameter Varying model where the contact quality coefficient is used as a scheduling parameter. Here, it is shown that monitoring the performance is not a good way for estimating the deterioration. The deterioration model, presented in this paper, is based on the dissipated energy at the contact level. The model allows to detect and to predict the moment of a possible outage of the actuator. Robustly positive invariant sets can be used to characterize the nominal behavior of the actuator and the maximal admissible deterioration behavior. This aspect can be used for starting decision-making activities related to actuator maintenance and/or control reconfiguration/replaning. However, this invariant sets are not enough for predicting the remaining useful life. A preliminary work using the Probabilistic certification approach has been presented and will be, combined with invariant sets, the object of a future work.

\section{REFERENCES}

[1] Mazen Alamir. On probabilistic certification of combined cancer therapies using strongly uncertain models. Journal of Theoretical Biology (Elsevier), 384:59-69, 2015.

[2] T. Alamo, R. Tempo, and E. F. Camacho. Randomized strategies for probabilistic solutions of uncertain feasibility and optimization problems. IEEE Transactions on Automatic Control, 54(11):25452559, Nov 2009.

[3] Adam Bevan, Paul Molyneux-Berry, Bridget Eickhoff, and Mark Burstow. Development and validation of a wheel wear and rolling contact fatigue damage model. Wear, 307(1-2):100 - 111, 2013.

[4] Marzia A. Cremona, Binbin Liu, Yang Hu, Stefano Bruni, and Roger Lewis. Predicting railway wheel wear under uncertainty of wear coefficient, using universal kriging. Reliability Engineering \& System Safety, 154:49 - 59, 2016.

[5] Carlos Canudas de Wit, Panagiotis Tsiotras, Efstathios Velenis, Michel Basset, and Gerard Gissinger. Dynamic friction models for road/tire longitudinal interaction. Vehicle System Dynamics, 39(3):189-226, 2003.

[6] Martinez J., Avila J., and Canudas de Wit C. A new bicycle vehicle model with dynamic contact friction. In IFAC Advances in Automotive Control, pages 625-630, Salerno, Italy, 2004. Elsevier.

[7] E.J. Kofman, H. Haimovich, and M.M. Seron. A systematic method to obtain ultimate bounds for perturbed systems. International Journal of Control, 80(2):167-178, February 2007.

[8] J.J. Martinez. Minimal RPI sets computation for polytopic systems using the bounded-real lemma and a new shrinking procedure. In 1st IFAC Workshop on Linear Parameter Varying systems (IFAC LPVS), Oct 2015.

[9] J.J. Martínez, M.M. Seron, and J.A. De Doná. Multi-sensor longitudinal control with fault tolerant guarantees. In Proceedings of the European Control Conference, Budapest - Hungary., September 2009.

[10] Tobias Meyer and Walter Sextro. Closed-loop control system for the reliability of intelligent mechatronic systems. In ASME, editor, European Conference of the Prognostics and Health Management Society 2014, number 265, pages 53-57, Paderborn, Germany, 2014.

[11] S.V. Rakovic, E.C. Kerrigan, K.I. Kouramas, and D.Q. Mayne. Invariant approximations of the minimal robust positively invariant set. IEEE Trans. on Automatic Control, 50(3):406 - 410, march 2005.

[12] Maria M. Seron, Xiang W. Zhuo, Jose A. De Dona, and John J. Martinez. Multisensor switching control strategy with fault tolerance guarantees. Automatica, 44(1):88 - 97, 2008.

[13] Xiao-Sheng Si, Wenbin Wang, Chang-Hua Hu, and Dong-Hua Zhou. Remaining useful life estimation-a review on the statistical data driven approaches. European Journal of Operational Research, 213(1):1-14, 2011. 